OPEN ACCESS

Edited by:

Wen Zhou,

Case Western Reserve University,

United States

Reviewed by:

Rosario Le Moli,

University of Catania, Italy

Kouki Mori,

JR Sendai Hospital, Japan

*Correspondence:

Angelo Cangiano

angelo.cangiano@unicampania.it

Specialty section:

This article was submitted to

Cancer Endocrinology,

a section of the journal

Frontiers in Endocrinology

Received: 23 July 2020

Accepted: 15 October 2020

Published: 04 December 2020

Citation:

Docimo G, Cangiano A, Romano RM,

Pignatelli MF, Offi C, Paglionico VA,

Galdiero M, Donnarumma G,

Nigro V, Esposito D, Rotondi M,

Candela $G$ and Pasquali D (2020)

The Human Microbiota in

Endocrinology: Implications for

Pathophysiology, Treatment, and

Prognosis in Thyroid Diseases.

Front. Endocrinol. 11:586529.

doi: 10.3389/fendo.2020.586529

\section{The Human Microbiota in Endocrinology: Implications for Pathophysiology, Treatment, and Prognosis in Thyroid Diseases}

\author{
Giovanni Docimo ${ }^{1}$, Angelo Cangiano ${ }^{1 *}$, Roberto Maria Romano ${ }^{1}$, \\ Marcello Filograna Pignatelli ${ }^{1}$, Chiara Offi ${ }^{1}$, Vanda Amoresano Paglionico ${ }^{2}$, \\ Marilena Galdiero ${ }^{3}$, Giovanna Donnarumma ${ }^{3}$, Vincenzo Nigro ${ }^{4}$, Daniela Esposito ${ }^{5}$, \\ Mario Rotondi ${ }^{6}$, Giancarlo Candela ${ }^{1}$ and Daniela Pasquali ${ }^{2}$ \\ ${ }^{1}$ Division of Thyroid Surgery, Department of Medical and Advanced Surgical Sciences, University of Campania "Luigi \\ Vanvitelli", School of Medicine, Naples, Italy, ${ }^{2}$ Department of Advanced Medical and Surgical Sciences, University of \\ Campania "Luigi Vanvitelli", Naples, Italy, ${ }^{3}$ Department of Experimental Medicine, University of Campania "Luigi Vanvitelli", \\ Naples, Italy, ${ }^{4}$ Department of Precision Medicine, University of Campania "Luigi Vanvitelli", Naples, Italy, ${ }^{5}$ Department of \\ Endocrinology, Institute of Medicine, Sahlgrenska Academy, University of Gothenburg and Sahlgrenska University Hospital, \\ Gothenburg, Sweden, ${ }^{6}$ Istituti Clinici Scientifici Maugeri IRCCS, Unit of Internal Medicine and Endocrinology, Laboratory for \\ Endocrine Disruptors, University of Pavia, Pavia, Italy
}

The human microbiota is an integral component in the maintenance of health and of the immune system. Microbiome-wide association studies have found numerous diseases associated to dysbiosis. Studies are needed to move beyond correlations and begin to address causation. Autoimmune thyroid diseases (ATD) are one of the most common organ-specific autoimmune disorders with an increasing prevalence, higher than 5\% worldwide. Most frequent manifestations of ATD are Hashimoto's thyroiditis and Graves' disease. The exact etiology of ATD remains unknown. Until now it is not clear whether bacterial infections can trigger ATD or modulate the efficacy of treatment and prognosis. The aim of our review is to characterize the microbiota and in ATD and to evaluate the impact of dysbiosis on treatment and prognosis. Moreover, variation of gut microbiome has been associated with thyroid cancer and benign nodules. Here we will characterize the microbioma in benign thyroid nodules, and papillary thyroid cancer to evaluate their implications in the pathophysiology and progression.

Keywords: gut microbiota, thyroid, cancer, autoimmunity, Hashimoto autoimmune thyroiditis

\section{GUT MICROBIOMA}

A lot of microbes, like bacteria, fungi, archaea, viruses, and protozoa, colonize our body. Many of these reside within the gastrointestinal tract and moreover on the ocular surface (1); they are predominantly bacterial, and together these microbes collectively form the gut microbiota. Gut microbiota contains trillions of microorganisms, about $66 \%$ of the human microbiota, including at least one thousand different species of known bacteria with more than 3 million genes (much more times than human genes). Thirty-three percent of these is common to all people, while $66 \%$ are 
explicit to every single one of us. This microbial flora has been coevolute with human being in a symbiotic relationship for millennia and, thusly, it finds itself involved in many essential activities for our organism such as digestion and nutritional absorption (2-4), development of the host's immunity system (3), and pathogenesis $(5,6)$. The gut microbiota was found to play an important role in maintaining the nutritional, metabolic, and immunological balance in the host (7). In addition to its intended role in maintaining gastrointestinal homeostasis, it also performs metabolic functions in digestion and nutrient absorption, detoxification, and vitamin synthesis. Furthermore, the microbiota also appears to be an important factor in the development of the lymphoid system, $70 \%$ of which resides in the intestinal mucosa. In the literature there are various examples, both on mouse models and on humans, which demonstrate an altered composition of the intestinal microbiota in a number of different pathological disorders, including obesity, compared to the "physiological" microbiota. For this reason, a substantial part of the studies on the gut microbiota treated so far has been dedicated to this topic. Interest in the role of the microbiota in health has increased, as evidenced by the May 2016 announcement of the National Microbiome Initiative (NMI) to promote the use of microbiota science in health care, but also for environmental restoration and food production. The new genome resource increases the capability to identify gut metagenomes over $87 \%(8,9)$. A recent study by Huiting et al. showed that iodine treatment affected the modulation mechanisms of thyroid function and the gut microbiota in obese mice. Administration of $\mathrm{KIO} 3$ in these animals led to weight reduction, increased concentration of thyroid hormones, alteration in the expression of the genes involved in thyroid biosynthesis, and cause various effects on the gut microbiota, changing the composition of the intestinal microenvironment, resulting in an imbalance of gut microbes: increases in pathogenic bacteria (Enterococcus, Clostridium, Fusobacterium nucleatum, Burkholderiales, Helicobacter), decrease in beneficial ones (Lactobacilli, Bifidobacteria), quite the contrary to what has been observed in non-obese hosts ( $\mathrm{p}<$ 0.05). Therefore, even with iodine dosages considered safe, obesity and quantitative and qualitative changes in the microbiota can increase the risk of thyroid dysfunction (10). Frohlich and Wahl concluded in their review that the composition of the microbiota and the different representation of its individual components in the various parts of the gastrointestinal system influence the absorption of $\mathrm{I}^{-}$, also modifying the enterohepatic circulation of thyroid hormones. Moreover, minerals such as $\mathrm{Se}, \mathrm{Fe}$, and $\mathrm{Zn}$ seem to be involved in the interaction between microbiota and host (11). The human microbiota seems to be a key regulator of health and diseases and the relevance of its influence on human health is gradually emerging. Gut dysbiosis is an alteration of the microbiota in its physiological function in the gastrointestinal tract; this condition can lead to local phlogosis and alteration of metabolic functions (12). In the gut dysbiosis there's a low microbial diversity (13) that is related with an extended range of human diseases, including changes in host immune status (14), asthma, allergies, inflammatory bowel disease (15-18), irritable bowel syndrome (19), obesity (20), chronic kidney disease (21), cardiovascular disease (22), and changes in blood pressure regulation (23). Additionally, alterations in gut microbial composition or function have been associated with age-related health impairment $(24,25)$. However, it's still difficult to establish the connections between the dysbiosis and these conditions, except for few cases. Furthermore, also changes in the viral component of microbiome (virome) can be associated with infectious and inflammatory diseases. Intestinal eukaryotic viruses have been implicated in triggering human type 1 diabetes mellitus (T1D). Changes in the intestinal virome, in particular Circoviridae-related sequences, have been found in who developed serum autoantibodies associated with T1D, suggesting that changes in the intestinal virome preceded autoimmunity (26). Some viruses establish subclinical lifelong persistent or latent infections in their host thereby becoming part of the normal microbiome. Human viral ecology is poorly understood yet. Paul G. Cantalupo et al. have developed a virus detection and discovery computational pipeline, Pickaxe, and applied it to NGS databases provided by The Cancer Genome Atlas (TCGA). They analyzed a collection of whole genome (WGS), exome (WXS), and RNA (RNA-Seq) sequencing libraries from 3,052 participants across 22 different cancers. HBV was also detected only in one sample of thyroid cancer, but these $\mathrm{HBV}$ sequences were due to sample cross contamination during sequencing, so they need to be confirmed. Moreover, viruses have long been considered potential triggers of autoimmune diseases (27).

\section{GUT MICROBIOME AND AUTOIMMUNITY}

The relation between autoimmunity and other systemic diseases has been for a long time examined by worldwide literature (2830 ), moreover focusing on new therapeutic implications in both surgical and medical field (31-38). The pathogenesis of autoimmune disorders is due to genetic (4-7, 23-25, 39-41), stochastic (42), and environmental (43-49) factors. In the environmental factors that have gained attention during the last decade, there is the intestinal microbiota. Several studies have supported a connection of altered microbiota composition with the beginning of several different autoimmune disorders, suggesting its role in the pathogenesis. These include Type I diabetes $(45,49)$; rheumatoid arthritis (50-53); systemic lupus erythematous $(54,55)$; inflammatory bowel disease comprising Cohn's disease and ulcerative colitis $(19,56)$; Bechet's disease (57); autoimmune skin conditions including vitiligo (58), atopic dermatitis (59, 60); psoriasis vulgaris (61), and autoimmune neurological diseases $(62,63)$. About $5-10 \%$ of the variability of bacterial taxa ascertained among individuals should be explained by genetic. Among the bacterial taxa transmitted, most of these is linked to genes involved in innate immunity (64). It is known that gastrointestinal microbiota is in reciprocal relationship with the host's immune system: immune system, through an equilibrium of pro- and anti-inflammatory pathways, 
has an important role in the modulation of the microbiome community; on the other hand microbiome has a critical role in the development of the immune system (65).

\section{MICROBIOME AND THYROID AUTOIMMUNE DISEASES}

Autoimmune thyroid disease (ATD) is a common autoimmune disorder. It's an organ-specific disorder and its prevalence is higher than 5\% worldwide and still increasing (66). The principal manifestation of the ATD are Graves' Disease (GD) and Hashimoto's Thyroiditis (HT). In HT there's a destruction of the thyroid cells that causes hypothyroidism, often detected in a subclinical condition (67), while in GD autoantibodies bind thyreotropin receptors stimulating an excessive production of thyroid hormone leading to hyperthyroidism. The role of $\mathrm{T}$ lymphocytes and their cytokines is indispensable for the immune modulation, but it's complex and full of connections with other components of the immunity. The correlation between gastrointestinal microbiota and development and progression of ATD has not yet been fully clarified. Nowadays, the analysis of reciprocal influence between microbiome and HT is a topic of considerable interest in the literature, since HT is the most frequent autoimmune disorder worldwide. There are several studies, both in animal models and in human, suggesting a link between modification in microbiota with the origins and development of ATD showing its key role in the thyroid peripheral homeostasis. Masetti et al. conducted a randomized controlled study on mouse models of GD. They studied the gut microbiota, observing a difference in biodiversity, spatial organization, and amount between the THSR immunized treated group and the untreated control group. The THSR immunized group developed signs of ophthalmopathy and their gut microbiota had more Firmicutes and less Bacteroides when comparing controls (68). However, already in 1988, Penhale and Young (69) noticed that modulation of the intestinal microbiota, in murine models affected by autoimmune thyroiditis, consistently made them more or less sensitive to thyroid autoimmunity. Lately, Köhling et al., through use of a PCR-denaturing gradient gel electrophoresis with universal primers targeting $\mathrm{V} 3$ region of the $16 \mathrm{~S}$ rRNA gene and quantitative real-time PCR, showed, in subjects affected by hyperthyroidism when compared with hypothyroid patients, an important difference of the intestinal microbiota composition, especially an overgrowth of bacteria in the small intestine, assessed by breath test with hydrogen glucose (70). Ishaq et al. found that there is a significant disparity between the gastrointestinal microbiota of patients affected by HT and healthy controls $(\mathrm{p}<0.05)$. Particularly, they showed raised level of Actinobacteria in HT group as compared to control. Moreover, levels of Prevotellaceae and Veillonellaceae were lower in diseased group if compared with healthy control, as well as Bifidobacterium and Lactobacillus; Veillonellaceae are commensal bacteria with an important role in regulation of adaptive immunity. At last, Enterobacteriaceae and Alcaligenaceae were higher in HT group as compared to control. Shigella and Escherichia can cause a broad spectrum of severe infectious diseases from hemorrhagic colitis to septicemia. In this paper, there's a clear demarcation of intestinal microbiota texture between HT patients and healthy group. The authors hypothesize that the raised levels of autoantibodies in these subpopulations might modify the structure of gastrointestinal microbiota, but other studies are obviously necessary to comprehend the underlying pathogenesis (71). Zhao F. et al. found similar and various concentrations of bacterial in the bowel microbiome of patients suffering from HT and controls $(\mathrm{p}=0.11)$. This study showed that levels of Blautia, Roseburia, Ruminococcus_torques_group, Romboutsia, Dorea, Fusicatenibacter, and Eubacterium_hallii_group were higher in patients affected by HT, while Fecalibacterium, Bacteroides, Prevotella_9, and Lachnoclostridium genera were lower in the same patients. Additionally, through the LEfSe method the authors were able to show many differences in these 27 genera composition between the patients and the healthy control, differences that were also correlated with clinical manifestation and laboratory data (72). Both in HT and GD the first therapy strategy is represented by the recovery of euthyroidism, in the first case by hormone replacement therapy, in the second case trough specific antithyroid drugs (73). The achievement of therapeutic goal may significantly differ, in both conditions, from patients to patients. Many factors can have a role: from age, sex, changes in BMI, to other conditions such as the thyroid hormones levels and the cause itself of the hypothyroidism, or the contemporary assumption of other drugs, some sorts of food and drink, soy protein, gastrointestinal malabsorption diseases and infections, bariatric surgery, atrophic gastritis, cystic fibrosis (74). All these parallel situations may influence the dosage of L-T4 replacement therapy in case of hypothyroidism and the severity of hyperthyroidism conditions in case of GD patients under antithyroid treatments. A disadvantage of antithyroid therapy is the high frequency of disease recurrence after the drug has been interrupted; these events occur often in the first year after discontinuing therapy, mostly in the first 6 months (75). In literature we aren't able to find many evidences about a possible correlation between gut microbiota changes and response to drug therapy. Actual studies have improved our knowledge about human microbiota, its basic functions and dynamics. The characterization of specific subset of bacterial species in ATD could help to identify new therapeutic strategy, by using probiotics, to rapidly gain therapeutic goal. Probiotics are available in many formulations, i.e. in the form of fermentable food, powders, or liquid drops. Many evidences show that probiotics, when given in the just dosage and for an adequate period of time, may lead to beneficial effects to human health; moreover they are often safer than many drugs. Probiotic impact on the immunity is important to comprehend how to therapeutically approach may interfere to the global increasing incidence of autoimmune diseases. INDIGO performed a doubleblind, placebo-controlled, randomized clinical study on the effects of a LAB4 probiotic on gut microbiota composition in patients with GD. There was a significant reduction in the Firmicutes phylum count in the treated group compared to placebo $(\mathrm{P}=0.033)$ and a temporary although important reduction in circulating autoantibodies and, consequently, in relapses at 6 months after antithyroid therapy, indicating the systemic immunomodulating effect of probiotics (76). 


\section{MICROBIOTA AND CANCER}

Some studies have shown the correlation of microbiota with cancer (77). Dysbiosis could initiate inflammatory and procarcinogenicity; conversely gut-derived probiotics restore gut commensal bacteria and protect the host including cancer disease, returning to a condition of wellness. Vivarelli et al. found that microbiota interferes directly host's DNA replication and its integrity. He shows ways of how pathogenic bacteria are capable to promote oncogenesis through the modulation or interfering with specifics host's oncogenic cell pathway or by interposing either with the hormonal or the host's immune system. Microbiota changes can release toxins that induce human DNA damage, concurring to its mutability, tumor induction and progression in gastrointestinal cancer. The author continues saying that "gut pathogenic bacteria can also disturb with DNA damage response and repair pathways, as in the case of Shigella flexneri, stimulating host's cells p53 degradation via the secretion of its enzymes inositol phosphate phosphatase $D(\mathrm{IpgD})$ and cysteine protease-like virulence gene A." Thus, it can increase the risk to induce mutations during the DNA damage response in infected cells. Cytotoxin associated gene A from Helicobacter pylori, causes the proteasome-mediated degradation of p53 in gastric epithelial cells, by interfering with the host's AKT pathway and inducing the gastric cancer. It is very complicated to determine in a clear way whether dysbiosis might have effects on the genesis of cancer or not. Additionally, changes in the everyday lifestyle, diet, and immune system factors, which deeply affect the microbiota composition and activity, may affect cancer genesis (78). Currently, Thyroid Carcinoma (TC) is the fifth most common malignancy diagnosed in female gender and its incidence is United States has increased an average of $3 \%$ per year in the last 4 decades; this increase is resulted by interactions among environmental factors, lifestyle, and genetic. Bowel microbiota, maybe, is an important environmental factor in gastrointestinal and extraintestinal tumor pathogenesis. However, regarding composition of bowel microbiome in patient affected by TC, there's limited information. Two categories include the contribution of gut microbiota to tumor pathogenesis. The first category includes the damage to DNA and apoptosis; bacteria like E. coli and Bacteroides fragilis may influence the host genomic stability leading to DNA damage and mutations, having and important role to colorectal carcinogenesis. The second category includes inflammatory reactions; many pattern recognitions receptors, comprehending Toll-Like Receptors (TLRs) are activated by cancer-associated microbial communities stimulating in loop $N F-k B$ signaling activation in the tumor microenvironment. Moreover, tumorigenesis obviously is influenced by specific pathogens and metabolic outputs of the gut microbiota. However, it has become increasingly clear that the collective activities of resident gut microbiota, particularly their metabolic products, strongly influence the protection against and predisposition to the development of malignancies. Up to now, there are only two reports in the literature, regarding the characterization of microbiota between healthy subjects, thyroid benign nodules and thyroid cancer patients. Feng et al. evaluated relationships among gastrointestinal microbiota, fecal metabolites, and TC. Through their researches they have noticed significative differences in gastrointestinal microbial communities from patients affected by TC and not affected ones, and also to the high or low quantity of bacteria's genera in TC, by using Ultraperformance liquid chromatography profiling offecal samples. In fecal metabolites composition were also identified many differences, indeed 72 metabolites showed important changes. Patients affected by TC were successfully identified from a combination of eight metabolites and five genera. Moreover, the findings implicate alterations in gut microbiota and metabolites in TC pathogenesis (79). In a recent cohort study by Zhang et al., the intestinal microbiota was compared with endocrine thyroid function both in patients with thyroid cancer and in subjects with benign nodular thyroid disease. The results made evident a difference in the microbiota between the thyroid pathology, both benign and malignant, and the healthy population: in particular, it was noticed a significant higher level of Neisseria and Streptococcus in patients with thyroid diseases while Butyricimonas underwent a significant decrease in patients with thyroid cancer and the same happened for Lactobacillus in patients with thyroid nodules $(\mathrm{p}<0.001)$. This shows that both pathological situations, benign and malignant thyroid diseases, correlate with the composition of the gut microbiota and consequently poses the possibility of any future treatments with specific probiotics (80). No data are present in the literature about the characterization of intestinal virome in thyroid cancer and thyroid nodules. Data collected over the past decade have identified the gut microbiota as an important factor defining inter individual variation in diseases risk. Evidence shows that gut microbiota and metabolites alter and potentially control cancerogenesis and progression.

\section{CONCLUSION}

Microbiome-wide association studies have established that numerous diseases are associated with changes in the microbiota. These studies typically generate a long list of commensals implicated as biomarkers of disease, with no clear relevance to disease pathogenesis. A major challenge is to understand which of the many diseases, including inflammatory diseases and various forms of cancer and cancer risk factors are detectable as different locations on this map. Our mini review tried to evaluate the correlation between thyroid diseases and gut dysbiosis, demonstrating that a changing in quality and quantity of intestinal commensal and pathogenic bacteria is associated with development of thyroid endocrine disease such as Hashimoto Thyroiditis and Graves' Disease as well as thyroid carcinoma. Future investigations are needed to identify the optimal probiotic and dose for specific diseases. The future of these researches is represented by the need to find the strict and direct correlation between the microbial changes and the specific pathology in order to identify the most targeted therapy possible, customized on each patient. 


\section{AUTHOR CONTRIBUTIONS}

All authors contributed significantly to the present research and reviewed the entire manuscript. GDoc: Participated substantially in conception and design of the manuscript and in the analysis and interpretation of the data. AC: Participated substantially in

\section{REFERENCES}

1. Petrillo F, Pignataro D, Lavano MA, Santella B, Folliero V, Zannella C, et al. Current Evidence on the Ocular Surface Microbiota and Related Diseases. Microorganisms (2020) 8(7):1033. doi: 10.3390/microorganisms8071033

2. Hehemann JH, Correc G, Barbeyron T, Helbert W, Czjzek M, Michel G. Transfer of carbohydrate-active enzymes from marine bacteria to Japanese gut microbiota. Nature (2010) 464(7290):908-12. doi: 10.1038/nature08937

3. Turnbaugh PJ, Gordon JI. The core gut microbiome, energy balance and obesity. J Physiol (2009) 587(Pt 17):4153-8. doi: 10.1113/jphysiol.2009.174136

4. Relman DA. The human microbiome, ecosystem resilience and health. Nutr $\operatorname{Rev}$ (2012) 70(Suppl 1):S2-9. doi: 10.1111/j.1753-4887.2012.00489.x

5. Baldridge MT, Nice TJ, McCune BT, Yokoyama CC, Kambal A, Whea- don $\mathrm{M}$, et al. Commensal microbes and interferon-lambda determine persistence of enteric murine norovirus infection. Science (2015) 347(6219):266-9. doi: $10.1126 /$ science. 1258025

6. Geddes K, Rubino SJ, Magalhaes JG, Streutker C, Le Bourhis L, Cho JH, et al. Identification of an innate $\mathrm{T}$ helper type 17 response to intestinal bacterial pathogens. Nat Med (2011) 17(7):837-44. doi: 10.1038/nm.2391

7. Dachao L, Ross Ka-Kit L, Wenda G, William WA. Involvement of gut microbiome in human health and disease, brief overview, knowledge gaps and research opportunities. Gut Pathog (2018) 10:3. doi: 10.1186/s13099-0180230-4

8. Sinha R, Ahsan H, Blaser M, Caporaso JG, Carmical JR, Chan AT, et al. Next steps in studying the human microbiome and health in prospective studies, Bethesda. Microbiome (2018) 6(1):210. doi: 10.1186/s40168-018-0596-Z

9. Pasolli E, Asnicar F, Manara S, Zolfo M, Karcher N, Armanini F, et al. Extensive Unexplored Human Microbiome Diversity Revealed by Over 150,000 Genomes from Metagenomes Spanning Age, Geography, and Lifestyle. Cell (2019) 176(3):649-62. doi: 10.1016/j.cell.2019.01.001

10. Huiting S, Jiaojiao H, Yanyan L, Chenyang L, Jun Z, Ye L, et al. Different hostspecific responses in thyroid function and gut microbiota modulation between diet-induced obese and normal mice given the same dose of iodine. Appl Microbiol Biotechnol (2019) 103(8):3537-47. doi: 10.1007/s00253-019-09687-1

11. Fröhlich E, Wahl R. Microbiota and Thyroid Interaction in Health and Disease. Trends Endocrinol Metab (2019) 30(8):479-90. doi: 10.1016/ j.tem.2019.05.008

12. Petersen C, Round JL. Defining dysbiosis and its influence on host immunity and disease. Cell Microbiol (2014) 16(7):1024-33. doi: 10.1111/cmi.12308

13. Kriss M, Hazleton KZ, Nusbacher NM, Martin CG, Lozupone CA. Low diversity gut microbiota dysbiosis, drivers, functional implications and recovery. Curr Opin Microbiol (2018) 44:34-40. doi: 10.1016/j.mib.2018.07.003

14. Rooks MG, Garrett WS. Gut microbiota, metabolites and host immunity. Nat Rev Immunol (2016) 16(6):341-52. doi: 10.1038/nri.2016.42

15. Carding S, Verbeke K, Vipond DT, Corfe BM, Owen LJ. Dysbiosis of the gut microbiota in disease. Microb Ecol Health Dis (2015) 26:26191. doi: 10.3402/ mehd.v26.26191

16. Arrieta MC, Stiemsma LT, Dimitriu PA, Thorson L, Russell S, Yurist-Doutsch S, et al. Early infancy microbial and metabolic alterations affect risk of childhood asthma. Sci Transl Med (2015) 7(307):ra152. doi: 10.1126/scitranslmed.aab2271

17. Bunyavanich S, Shen N, Grishin A, Wood R, Burks W, Dawson P, et al. Earlylife gut microbiome composition and milk allergy resolution. J Allergy Clin Immunol (2016) 138(4):1122-30. doi: 10.1016/j.jaci.2016.03.041

18. Nishino K, Nishida A, Inoue R, Kawada Y, Ohno M, Sakai S, et al. Analysis of endoscopic brush samples identified mucosa-associated dysbiosis in inflammatory bowel disease. J Gastroenterol (2018) 53(1):95-106. doi: 10.1007/s00535-017-1384-4 conception, design, and execution of the study and in the analysis and interpretation of the data; also participated substantially in the drafting and editing of the manuscript. RR, MF, CO, VA, MG, GDon, VN, MR, GC and DP: Participated substantially in conception and design of the manuscript and in the analysis and interpretation of the data. All the authors have read an approved the final manuscript.

19. Cao SS. Cellular Stress Responses and Gut Microbiota in Inflammatory Bowel Disease. Gastroenterol Res Pract (2018) 2018:7192646. doi: 10.1155/2018/ 7192646

20. Schwiertz A, Taras D, Schäfer K, Beijer S, Bos NA, Donus C, et al. Microbiota and SCFA in lean and overweight healthy subjects. Obes (Silver Spring) (2010) 18(1):190-5. doi: 10.1038/oby.2009.167

21. Sircana A, De Michieli F, Parente R, Framarin L, Leone N, Berrutti M, et al. Gut Microbiota, Hypertension and Chronic kidney Disease, recent advances. Pharmacol Res (2018) 144:390-408. doi: 10.1016/j.phrs.2018.01.013

22. Jie Z, Xia H, Zhong SL, Feng Q, Li S, Liang S, et al. The gut microbiome in atherosclerotic cardiovascular disease. Nat Commun (2017) 8(1):845. doi: 10.1038/s41467-017-00900-1

23. Marques FZ, Mackay CR, Kaye DM. Beyond gut feelings, how the gut microbiota regulates blood pressure. Nat Rev Cardiol (2018) 15(1):20-32. doi: 10.1038/nrcardio.2017.120

24. Clark RI, Walker DW. Role of gut microbiota in aging-related health decline, insights from invertebrate models. Cell Mol Life Sci (2018) 75(1):93-101. doi: 10.1007/s00018-017-2671-1

25. O’Toole PW, Jeffery IB. Microbiome-health interactions in older people. Cell Mol Life Sci (2018) 75(1):119-28. doi: 10.1007/s00018-017-2673-z

26. Zhao G, Vatanen T, Droit L, Park A, Kostic AD, Poon TW, et al. Intestinal virome changes precede autoimmunity in type I diabetes-susceptible children. Proc Natl Acad Sci USA (2017) 114(30):E6166-75. doi: 10.1073/pnas. 1706359114

27. Cantalupo PG, Katz JP, Pipas JM. Viral sequences in human cancer. Virology (2018) 513:208-16. doi: 10.1016/j.virol.2017.10.017

28. Pasquali D, Maiorino NII, Renzullo A, Bellastella G, Accardo G, Esposito D, et al. Female sexual dysfunction in women in thyroid disorders. J Endocrinol Invest (2013) 36:729-33. doi: 10.3275/8933

29. Balercia G, Bonomi M, Giagulli VA, Lanfranco F, Rochira V, Giambersio A, et al. Thyroid function in Klinefelter syndrome: a multicentre study from KING group. J Endocrinol Invest (2019) 42(10):1199-204. doi: 10.1007/ s40618-019/01037-2

30. Pasquali D, Notaro A, Bonavolontà G, Vassallo P, Bellastella A, Sinisi AA. Somatostatin receptor genes are expressed in lymphocytes from retroorbital tissues in Graves' Disease. J Clin Endocrinol Metab (2002) 87(11):5525-129. doi: $10.1210 /$ jc.2002-020790

31. Esposito D, Rotondi M, Accardo G, Vallone G, Conzo G, Docimo G, et al. Influcence of short-term selenium supplementation on the natural course of Hashimoto's Thyroiditis: clinical results of a blinded placebo-controlled randomized prospective trial. J Endocrinol Invest (2017) 40(1):83-9. doi: 10.1007/s40618-016-0535-4

32. Pirola I, Rotondi M, Cristiano A, Maffezzoni F, Pasquali D, Marini F, et al. Selenium supplementation in patients with subclinical hypothiroidism affected by autoimmune thyroiditis: Results of the SETI study. Endocrinol Diabetes Nutr (2020) 67(1):2835. doi: 10.1016/j.endien.2019.12.002

33. Croce L, De Martinis L, Pinto S, Coperchini F, Dito G, Bendotti G, et al. Compared with classic Hashimoto's Thyroiditis, chronic autoimmune serumnegative thyroiditis requires a lower substitution dose of L-thyroxine to correct hypothyroidism. J Endocrinol Invest (2020) 43(11):1631-6. doi: 10.1007/s40618-020-01249-x

34. Ruggiero R, Gubitosi A, Conzo G, Gili S, Bosco A, Pierozzi R, et al. Sutureless thyroidectomy. Int J Surg (2014) 12:189-93. doi: 10.1016/j.ijsu.2014.05.011

35. Docimo G, Tolone S, Pasquali D, Conzo G, D'Alessandro A, Casalino G, et al. Role of pre and post-operative oral calcium and vitamin D supplements in prevention of hypocalcemia after total thyroidectomy. J Surg (2012) 33(1112):974-8. 
36. Pezzolla A, Docimo G, Ruggiero R, Monacelli M, Cirocchi R, Parmeggiani D, et al. Incidental thyroid carcinoma: a multicentric experience. Recenti Prog Med (2010) 101(5):194-8. doi: 10.1701/493.5851

37. Docimo G, Tolone S, Ruggiero R, Gubitosi A, Pasquali D, De Bellis A, et al. Total thyroidectomy without profilattic central neck dissection combined with routine oral calcium and vitamine $\mathrm{D}$ supplement: Is it a good option to archieve a low recurrence rate avoiding hypocalcemia? A retrospective study. Minerva Chirurgica (2013) 68(3):321-8.

38. Docimo G, Ruggiero R, Casalino G, Del Genio G, Docimo L, Tolone S. Risk factors for postoperative hypocalcemia. Updates Surg (2017) 69(2):255-60. doi: 10.1007/s13304-017-0452-x

39. Ajayi T, Innes CL, Grimm SA, Rai P, Finethy R, Coers J, et al. Crohn's disease IRGM risk alleles are associated with altered gene expression in human tissues. Am J Physiol Gastrointest Liver Physiol (2019) 316:G95-G105. doi: 10.1152/ajpgi.00196.2018

40. Batura V, Muise AM. Very early onset IBD, Novel genetic aetiologies. Curr Opin Allergy Clin Immunol (2018) 18:470-80. doi: 10.1097/ACI.0000000000000486

41. Zhang J, Meng Y, Wu H, Wu Y, Yang B, Wang L. Association between PPP2CA polymorphisms and clinical features in southwest Chinese systemic lupus erythematosus patients. Medicine (2018) 97(27):e11451. doi: 10.1097/ MD.0000000000011451

42. Wu YL, Ding YP, Gao J, Tanaka Y, Zhang W. Risk factors and primary prevention trials for type 1 diabetes. Int J Biol Sci (2013) 9:666-79. doi: $10.7150 /$ ijbs. 6610

43. Barbeau WE. What is the key environmental trigger in type 1 diabetes-Is it viruses, or wheat gluten, or both? Autoimmun Rev (2012) 12:295-9. doi: 10.1016/j.autrev.2012.05.003

44. Lindoso L, Mondal K, Venkateswaran S, Somineni HK, Ballengee C, Walters TD, et al. The Effect of Early-Life Environmental Exposures on Disease Phenotype and Clinical Course of Crohn's Disease in Children. Am J Gastroenterol (2018) 113:1524-9. doi: 10.1038/s41395-018-0239-9

45. Gianchecchi E, Fierabracci A. On the pathogenesis of insulin-dependent diabetes mellitus, The role of microbiota. Immunol Res (2017) 65:242-56. doi: $10.1007 / \mathrm{s} 12026-016-8832-8$

46. Gianchecchi E, Fierabracci A. Recent Advances on Microbiota Involvement in the Pathogenesis of Autoimmunity. Int J Mol Sci (2019) 20(2):283. doi: 10.3390/ijms20020283

47. Henschel AM, Cabrera SM, Kaldunski ML, Jia S, Geoffrey R, Roethle MF, et al. Modulation of the diet and gastrointestinal microbiota normalizes systemic inflammation and $\beta$-cell chemokine expression associated with autoimmune diabetes susceptibility. PloS One (2018) 13(1):e0190351. doi: 10.1371/ journal.pone.0190351

48. Huang Y, Li SC, Hu J, Ruan HB, Guo HM, Zhang HH, et al. Gut microbiota profiling in Han Chinese with type 1 diabetes. Diabetes Res Clin Pract (2018) 141:256-63. doi: 10.1016/j.diabres.2018.04.032

49. Mullaney JA, Stephens JE, Costello ME, Fong C, Geeling BE, Gavin PG, et al. Correction to, Type 1 diabetes susceptibility alleles are associated with distinct alterations in the gut microbiota. Microbiome (2018) 6:51. doi: 10.1186/ s40168-018-0438-z

50. Sato K, Takahashi N, Kato T, Matsuda Y, Yokoji M, Yamada M, et al. Aggravation of collagen-induced arthritis by orally administered Porphyromonas gingivalis through modulation of the gut microbiota and gut immune system. Sci Rep (2017) 7:6955. doi: 10.1038/s41598-01707196-7

51. Teng F, Felix KM, Bradley CP, Naskar D, Ma H, Raslan WA, et al. The impact of age and gut microbiota on Th17 and Tfh cells in $\mathrm{K} / \mathrm{BxN}$ autoimmune arthritis. Arthritis Res Ther (2017) 19(1):188. doi: 10.1186/ s13075-017-1398-6

52. Jubair WK, Hendrickson JD, Severs EL, Schulz HM, Adhikari S, Ir D, et al. Modulation of Inflammatory Arthritis in Mice by Gut Microbiota Through Mucosal Inflammation and Autoantibody Generation. Arthritis Rheumatol (2018) 70:1220-33. doi: 10.1002/art.40490

53. Picchianti-Diamanti A, Panebianco C, Salemi S, Sorgi ML, Di Rosa R, Tropea A, et al. Analysis of Gut Microbiota in Rheumatoid Arthritis Patients, DiseaseRelated Dysbiosis and Modifications Induced by Etanercept. Int J Mol Sci (2018) 19:2938. doi: 10.3390/ijms19102938

54. Calderaro DC, Ferreira GA, de Mendonça SMS, Corrêa JD, Santos FX, Sanção JGC, et al. Há associação entre o lúpus eritematoso sistêmico e a doença periodontal? Rev Bras Reumatol (2016) 56:280-4. doi: 10.1016/j.rbr.2015. 03.011

55. Corrêa JD, Calderaro DC, Ferreira GA, Mendonça SM, Fernandes GR, Xiao E, et al. Subgingival microbiota dysbiosis in systemic lupus erythematosus, Association with periodontal status. Microbiome (2017) 5:34.

56. Ni J, Wu GD, Albenberg L, Tomov VT. Gut microbiota and IBD, Causation or correlation? Nat Rev Gastroenterol Hepatol (2017) 14:573-84. doi: 10.1038/ nrgastro.2017.88

57. Zi Y, Ni Z, Chunyan W, Xinyuan Z, Qingfeng W, Xinyue H, et al. A metagenomic study of the gut microbiome in Behcet's disease. Microbiome (2018) 6:135.

58. Ganju P, Nagpal S, Mohammed MH, Nishal Kumar P, Pandey R, Natarajan VT, et al. Microbial community profiling shows dysbiosis in the lesional skin of Vitiligo subjects. Sci Rep (2016) 13(6):18761. doi: $10.1038 /$ srep 18761

59. Kim MH, Choi SJ, Choi HI, Choi JP, Park HK, Kim EK, et al. Lactobacillus plantarum-derived Extracellular Vesicles Protect Atopic Dermatitis Induced by Staphylococcus aureus-derived Extracellular Vesicles. Allergy Asthma Immunol Res (2018) 10:516-32. doi: 10.4168/aair.2018.10.5.516

60. Suzuki S, Campos-Alberto E, Morita Y, Yamaguchi M, Toshimitsu T, Kimura K, et al. Low Interleukin 10 Production at Birth Is a Risk Factor for Atopic Dermatitis in Neonates with Bifidobacterium Colonization. Int Arch Allergy Immunol (2018) 11:1-8. doi: 10.1159/000492130

61. Chang HW, Yan D, Singh R, Liu J, Lu X, Ucmak D, et al. Alteration of the cutaneous microbiome in psoriasis and potential role in Th17 polarization. Microbiome (2018) 6:154. doi: 10.1186/s40168-018-0533-1

62. Berer K, Gerdes LA, Cekanaviciute E, Jia X, Xiao L, Xia Z, et al. Gut microbiota from multiple sclerosis patients enables spontaneous autoimmune encephalomyelitis in mice. Proc Natl Acad Sci USA (2017) 114:10719-24. doi: $10.1073 /$ pnas. 1711233114

63. Cekanaviciute E, Yoo BB, Runia TF, Debelius JW, Singh S, Nelson CA, et al. Gut bacteria from multiple sclerosis patients modulate human T cells and exacerbate symptoms in mouse models. Proc Natl Acad Sci USA (2017) 114:10713-8. doi: 10.1073/pnas.1711235114

64. Hall AB, Tolonen AC, Xavier RJ. Human genetic variation and the gut microbiome in disease. Nat Rev Genet (2017) 18(11):690-9. doi: 10.1038/ nrg. 2017.63

65. Vatanen T, Kostic AD, d'Hennezel E, Siljander H, Franzosa EA, Yassour M, et al. Variation in Microbiome LPS Immunogenicity Contributes to Autoimmunity in Humans. Cell (2016) 165(6):1551. doi: 10.1016/j.cell.2016.05.056

66. Iddah MA, Macharia BN. Autoimmune thyroid disorders. ISRN Endocrinol (2013) 26:509764. doi: 10.1155/2013/509764

67. Marfella R, Ferraraccio F, Rizzo MR, Portoghese M, Barbieri M, Basilio C, et al. Innate immune activity in plaque of patients with untreated and Lthyroxine-treated subclinical hypothyroidism. J Clin Endocrinol Metab (2011) 96(4):1015-20. doi: 10.1210/jc.2010-1382

68. Masetti G, Moshkelgosha S, Köhling HL, Covelli D, Banga JP, BerchnerPfannschmidt $U$, et al. Gut microbiota in experimental murine model of Graves' orbitopathy established in different environments may modulate clinical presentation of disease. Microbiome (2018) 6(1):97. doi: 10.1186/ s40168-018-0478-4

69. Penhale WJ, Young PR. The influence of the normal microbial flora on the susceptibility of rats to experimental autoimmune thyroiditis. Clin Exp Immunol (1988) 72(2):288-92.

70. Köhling HL, Plummer SF, Marchesi JR, Davidge KS, Ludgate M. The microbiota and autoimmunity, Their role in thyroid autoimmune diseases. Clin Immunol (2017) 183:63-74. doi: 10.1016/j.clim.2017.07.001

71. Ishaq HM, Mohammad IS, Guo H, Shahzad M, Hou YJ, Ma C, et al. Molecular estimation of alteration in intestinal microbial composition in Hashimoto's thyroiditis patients. BioMed Pharmacother (2017) 95:865-74. doi: 10.1016/ j.biopha.2017.08.101

72. Zhao F, Feng J, Li J, Zhao L, Liu Y, Chen H, et al. Alterations of the Gut Microbiota in Hashimoto's Thyroiditis Patients. Thyroid (2018) 28(2):175-86. doi: 10.1089/thy.2017.0395

73. Peirce C, Ippolito S, Lanas A, Pesce M, Pontieri G, Arpaia D, et al. Treatment of refractory and severe hypothyroidism with sublingual levothyroxine in liquid formulation. Endocrine (2018) 60(1):193-6. doi: 10.1007/s12020-017$1367-5$ 
74. Virili C, Antonelli A, Santaguida MG, Benvenga S, Centanni M. Gastrointestinal Malabsorption of Thyroxine. Endocr Rev (2019) 40(1):11836. doi: 10.1210/er.2018-00168

75. De Leo S, Lee SY, Braverman LE. Hyperthyroidism. Lancet (2016) 388 (10047):906-18. doi: 10.1016/S0140-6736(16)00278-6

76. Salvi M, Colucci G, Masetti G, Covelli D, Muller I, Koehling H, et al. The randomised probiotic trial of indigo study (investigation of novel biomarkers and definition of role of the microbiome in Graves' orbitopathy). Endocr Abstracts (2019) 63:GP71. doi: 10.1530/endoabs.63.GP71

77. Zhang YJ, Li S, Gan RY, Zhou T, Xu DP, Li HB. Impacts of gut bacteria on human health and diseases. Int J Mol Sci (2015) 16:7493-519. doi: 10.3390/ ijms 16047493

78. Vivarelli S, Salemi R, Candido S, Falzone L, Santagati M, Stefani S, et al. Gut Microbiota and Cancer, From Pathogenesis to Therapy. Cancers (2019) 11 (1):38. doi: 10.3390/cancers 11010038

79. Feng J, Zhao F, Sun J, Lin B, Zhao L, Liu Y, et al. Alterations in the gut microbiota and metabolite profiles of thyroid carcinoma patients. Int $J$ Cancer (2019) 144(11):2728-45. doi: 10.1002/ijc.32007
80. Zhang J, Fanghua Z, Zhang F, Zhao C, Xu Q, Liang C, et al. Dysbiosis of the gut microbiome is associated with thyroid cancer and thyroid nodules and correlated with clinical index of thyroid function. Endocrine (2019) 64:564-74 doi: 10.1007/s12020-018-1831-x

Conflict of Interest: The authors declare that the research was conducted in the absence of any commercial or financial relationships that could be construed as a potential conflict of interest.

Copyright (c) 2020 Docimo, Cangiano, Romano, Pignatelli, Offi, Paglionico, Galdiero, Donnarumma, Nigro, Esposito, Rotondi, Candela and Pasquali. This is an openaccess article distributed under the terms of the Creative Commons Attribution License (CC BY). The use, distribution or reproduction in other forums is permitted, provided the original author(s) and the copyright owner(s) are credited and that the original publication in this journal is cited, in accordance with accepted academic practice. No use, distribution or reproduction is permitted which does not comply with these terms. 